\title{
Normalización del cemento. Características químicas: Algunos comentarios sobre los métodos de ensayo
}

\author{
Prof. IOr. DEMETRIO GASPAR-TEBAR
}

IETCe

\section{INTRODUCCION}

El Pliego de Preseripciones Técnicas Generales para la Recepción de Cementos (RC-75), Decreto $1.064 / 1975$ de 23 de mayo de 1975, publicado en los B.O.E. núms. 206 y 207, de fechas 28 y 29 de agosto, respectivamente, del mismo año, incluye la clasificación de los cementos, las prescripciones relativas a las características químicas y físicas, así como los métodos de ensayo que se deben utilizar para determinar dichas características.

Los diversos ensayos que se realizan en la industria del cemento tienen por objeto fabricar un producto que responda a unas propiedades determinadas y proporcionar, además, unos datos de modo que los proyectistas y los constructores tengan a su alcance informaciones precisas que puedan ser útiles para el cálculo de las obras, para conocer su durabilidad probable y para poder elegir el cemento más apropiado en cada caso particular.

Los tipos de cemento que se establecen en el Pliego RC-75 son los siguientes:

- Portland.

- Portland con adiciones activas.

- Siderúrgico.

- Puzolánico.

- Compuesto.

- Aluminoso.

- Natural.

En este artículo se estudian los métodos de ensayo normalizados para determinar las características químicas de los cementos de uso más frecuente, es decir, de los cementos portland $(\mathrm{P})$, de los cementos portland con adiciones activas (PA), de los cementos siderúrgicos (S) y de los cementos puzolánicos (PUZ-I y PUZ-II).

\section{II: COMPOSICION DE LOS CEMENTOS}

Los cementos se caracterizan, fundamentalmente, por los componentes que forman el conjunto (cemento) y por la proporción de cada uno de ellos. En el nuevo Pliego RC-75 se han 
aceptado como cementos portland a mezclas binarias de clínker y regulador del fraguado (normalmente yeso); como cementos portland con adiciones activas a mezclas ternarias o cuaternarias de clínker de cemento portland, regulador del fraguado (normalmente yeso) y escoria siderúrgica o/y puzolana; como cementos siderúrgicos y como cementos puzolánicos a mezclas ternarias de clínker de cemento portland, regulador del fraguado (normalmente yeso) y escoria siderúrgica o puzolana, respectivamente.

Los cementos puzolánicos pueden contener bien puzolana natural o de cualquier otro tipo, excluidas las cenizas volantes (PUZ-I), o bien cenizas volantes (PUZ-II).

Como adiciones activas se pueden utilizar aquellos materiales que posean propiedades hidráulicas latentes, como algunas escorias siderúrgicas, o que sean capaces de fijar cal como las puzolanas naturales o artificiales (tierra de diatomeas, arcillas activadas y cenizas volantes).

La composición de los cementos de uso más frecuente ${ }_{\Downarrow}$ así como la proporción de los distintos componentes, en $\%$ en peso, figuran en la tabla 1.

$$
\text { TAB L A } 1
$$

Composición de los cementos normales, según el Pliego RC-75

\begin{tabular}{|c|c|c|c|c|c|}
\hline Tipo de cemento & $\begin{array}{c}\text { Clínker } \\
+ \\
\end{array}$ & $\begin{array}{l}\text { Adición } \\
\text { activa }\end{array}$ & Escoria & Puzolana (*) & $\begin{array}{c}\text { Cenizas } \\
\text { volantes }(* *)\end{array}$ \\
\hline Portland & 100 & - & - & - & - \\
\hline $\begin{array}{l}\text { Portland con adiciones } \\
\text { activas }\end{array}$ & $\geqslant 80$ & $\leqslant 20$ & - & - & - \\
\hline $\begin{array}{lc} & \text { I } \\
\text { Siderúrgico } & \text { II } \\
& \text { III }\end{array}$ & $\begin{array}{l}\geqslant 70 \mathrm{y}<80 \\
\geqslant 50 \mathrm{y}<70 \\
>20 \mathrm{y}<50\end{array}$ & $\begin{array}{l}- \\
- \\
-\end{array}$ & $\begin{array}{l}\leqslant 30 \mathrm{y}>20 \\
\leqslant 50 \mathrm{y}>30 \\
<80 \mathrm{y}>50\end{array}$ & $\begin{array}{l}- \\
- \\
-\end{array}$ & $\begin{array}{l}- \\
- \\
-\end{array}$ \\
\hline $\begin{array}{ll} & \text { I } \\
\text { Puzolánico } & \text { II }\end{array}$ & $\begin{array}{l}<80 \\
<80\end{array}$ & - & - & $\begin{array}{c}>20 \\
-\end{array}$ & $\begin{array}{l}- \\
>20\end{array}$ \\
\hline
\end{tabular}

(*) Puzolana natural o de cualquier otro tipo, excluidas las cenizas volantes; cementos puzolánicos (PUZ-I).

(**) Cementos puzolánicos (PUZ-II), con cenizas volantes.

Los cementos portland con adiciones activas, los cementos siderúrgicos y los cementos puzolánicos, según RC-75, se obtienen-por molturación conjunta del clínker de cemento portland, del regulador del fraguado y de la adición activa (algunas escorias siderúrgicas o/y puzolana) los primeros, de la escoria siderúrgica, los segundos y de la puzolana los terceros.

Los cementos portland se obtienen también por molturación conjunta de su clínker y del regulador del fraguado.

\section{Características químicas}

Las prescripciones relativas a las características químicas de los cementos señalados anteriormente, según RC-75, referidas a la muestra seca a $105-110^{\circ} \mathrm{C}$ y expresadas en $\%$ en peso, son las que se incluyen en la tabla 2 . 
TAB A A 2

Prescripciones relativas a las características químicas, según el Pliego RC-75

\begin{tabular}{|c|c|c|c|c|}
\hline & \multicolumn{3}{|c|}{ Tipo de cemento } \\
\cline { 2 - 5 } & Portland & $\begin{array}{c}\text { Portland } \\
\text { con adiciones } \\
\text { activas }\end{array}$ & Siderúrgico & Puzolánico \\
\hline $\begin{array}{c}\text { Pérdida al fuego } \\
\text { máximo, \% }\end{array}$ & 4 y 3,5 & 4 & 4 & - \\
\hline $\begin{array}{c}\text { Residuo insoluble } \\
\text { máximo, \% }\end{array}$ & 3 y 2,5 & $4\left(^{*}\right)$ & 3 & - \\
\hline $\begin{array}{c}\text { Oxido de magnesio } \\
\text { máximo, \% }\end{array}$ & 5 & 5 & 4,5 & 3,5 \\
\hline $\begin{array}{c}\text { Trióxido de azufre } \\
\text { máximo, \% }\end{array}$ & 4 y 4,5 & 4 & - \\
\hline
\end{tabular}

(*) Si la adición es de puzolana se eleva al $8 \%$ y si es de ceniza volante no se limits.

Para los cementos tipo portland y portland con adiciones activas se fija la pérdida al fuego o pérdida por calcinación, el residuo insoluble en ácido clorhídrico, con las salvedades que se indican en dicha tabla, el óxido de magnesio y el trióxido de azufre; sin embargo. para los cementos siderúrgicos se limita la pérdida al fuєgo o pérdida por calcinación, el residuo insoluble en ácido clorhídrico y el trióxido de azufre, y para los cementos puzolánicos se fija, únicamente, el contenido de trióxido de azufre.

\section{COMPORTAMIENTO DE LOS COMPONRNES DE LOS CEMENTOS (RC-75) FRENTE A DISOLUCIONES DE ACIDO CLORHIDRICO Y A LA ACCION DEL CALOR}

En este apartado se considera la acción de disaluciones de ácido clorhídrico y del calor sobre los componentes de los cementos reseñados (portland, portland con adiciones activas, siderúrgicos y puzolánicos) para contribuir al estudio de las técnicas de trabajo normalizadas en el Pliego RC-75.

\section{Clinker de cemento portland}

El clínker de cemento portland es el producto que se obtiene al calcinar hasta fusión parcial un crudo de cemento portland, durante el tiempo necesario, para conseguir la combinación prácticamente total de sus componentes (definición del Pliego RC-75).

El clínker está formado por fases cristalinas en gran proporción y vítreas en cantidad apreciable, que corresponden a compuestos cálcicos $\left(\mathrm{C}_{3} \mathrm{~S}, \mathrm{C}_{2} \mathrm{~S}, \mathrm{C}_{3} \mathrm{~A}, \mathrm{C}_{4} \mathrm{AF}\right)$ solubles en ácido clorhídrico. Cuando el clínker bien cocido se ataca con $\mathrm{HCl}$ (disolución acuosa) suele quedar un residuo insoluble que, una vez tratado con una disolución de $\mathrm{Na}_{2} \mathrm{CO}_{3}$, es menor del $0,5 \%$, el cual procede de las materias primas del crudo, normalmente de tipo arcilloso, que por su naturaleza y características físicas no reaccionaron durante el proceso de clinkerización. 
La determinación del residuo insoluble (ataque con una disolución de $\mathrm{HCl}$ y tratamiento del residuo, posteriormente, con otra disolución de $\mathrm{Na}_{2} \mathrm{CO}_{3}$ ) de crudos calcinados en el laboratorio, a diferentes temperaturas, se toma como un procedimiento para medir el grado de clinkerización y para determinar la aptitud de dichos crudos a la calcinación.

Los compuestos de magnesio (II) que se encuentran formando parte de las fases principales del clínker y como $\mathrm{MgO}\left({ }^{*}\right)$, asi como los correspondientes a los sulfatos (**), que se pueden formar según se señala a continuación, son solubles en la disolución ocuosa de $\mathrm{HCl}$ por lo que se encuentran, como iones, en la fase líquida.

Los combustibles utilizados en la industria del cemento contienen cantidades variables de azufre (***) que durante la combustión se oxida a $\mathrm{SO}_{2}$, el cual se combina con los álcalis volatilizados que se encuentran también en los gases del horno, produciendo sulfatos alcalinos, una vez transformado el $\mathrm{SO}_{2}$ en $\mathrm{SO}_{3}$ por la acción del oxígeno del aire en presencia de catalizadores, como es el $\mathrm{Fe}_{2} \mathrm{O}_{3}$. Estos sulfatos se depositan, en parte, en las zonas frías del horno y en el intercambiador; una fracción se elimina en el polvo de la chimenea. Los sulfatos alcalinos condensados retornan a la zona de clinkerización incorporándose al clínker.

Cuando el $\mathrm{SO}_{2}$ se encuentra en exceso puede tener lugar otra reacción con el $\mathrm{CaCO}_{3}$, en el intercambiador, dando $\mathrm{CaSO}_{4}$ que vuelve a la zona de clinkerización, en donde experimenta parcialmente la disociación térmica; el $\mathrm{SO}_{3}$ se incorpora a los gases de combustión y el resto del $\mathrm{CaSO}_{4}$ no disociado queda en el clínker.

Un clínker bien cocido no debe experimentar, teóricamente, variación de masa cuando se sometes a la acción del calor en atmósfera oxidante; el intervalo de temperaturas especificado en el Pliego RC-75 para determinar la pérdida al fuego del cemento es $900-1.000^{\circ} \mathrm{C}$. Por regla general se produce una pérdida de masa debida a la disociación térmica que experimentan los compuestos que, en gran parte y en pequeña proporción $(* * * *)$, se han formado durante la meteorización que pueden experimentar las partículas del clínker molido, por tratarse de un material pulverulento, muy fino y activo, sensible a la humedad y al dióxido de carbono presentes en la atmósfera.

A veces aparecen compuestos reductores en los clínkeres, sobre todo en los obtenidos en hornos verticales; estos compuestos pueden dar lugar, durante la calcinación en atmósfera oxidante, a la formación de otros de mayor masa, hecho que influye en la pérdida de masa del conjunto por tratarse de un fenómæno aditivo.

\section{Regulador del fraguado}

El Pliego RC-75 define como regulador del fraguado al producto que añadido al clínker de cemento portland en cantidad adecuada y molido conjuntamente con él, proporciona un cemento de fraguado normal. Generalmente se utiliza el sulfato de calcio en alguna de sus variedades o mezcla de ellas.

En la fabricación de cemento portland se utiliza el yeso natural, variedad que cristaliza con dos moléculas de agua $\left(\mathrm{CaSO}_{4} \cdot 2 \mathrm{H}_{2} \mathrm{O}\right)$ y/o la anhidrita $\left(\mathrm{CaSO}_{4}\right)$. El hemihidrato $\left(2 \mathrm{CaSO}_{4} \cdot \mathrm{H}_{2} \mathrm{O}<>\mathrm{CaSO}_{4} \cdot 1 / 2 \mathrm{H}_{2} \mathrm{O}\right)$ puede producirse, sin propósito previo, durante la molienda del cemento o, menos frecuentemente, durante su almacenamiento.

El yeso natural o aljez, cuando está puro, tiene $79,1 \%$ de $\mathrm{CaSO}_{4}(32,6 \%$ de $\mathrm{CaO}$ y $46,5 \%$ de $\mathrm{SO}_{3}$ ) y $20,9 \%$ de $\mathrm{H}_{2} \mathrm{O}$; ahora bien, el yeso natural se encuentra contaminado por impurezas tales como arcilla, caliza, dolomía, compuestos de hierro, sílice, etc.

\footnotetext{
(*) Hasta el $2 \%$ en las fases principales del cínker y como MgO por encima de esta cantidad.

(**) El contenido medio de sulfatos es del orden de 0,6\%.

(***) Desde $0 \%$ para el gas natural hasta $3,5 \%$ para el fuel-oil pesado.

$(* * * *) \quad$ Normalmente es menor del $1 \%$.
} 
La anhidrita natural pura contiene 58,8 \% de $\mathrm{SO}_{3}$ y $41,2 \%$ de $\mathrm{CaO}$; no tiene agua de cristalización, pero es capaz de fijar agua y transformarse en yeso; este cambio se favorece conforme disminuye el tamaño del grano.

Tanto el yeso como la anhidrita son ligeramente solubles en agua $(0,223$ partes de yeso en 100 partes de agua, a $0^{\circ} \mathrm{C}$, y 0,257 partes de yeso, a $50^{\circ} \mathrm{C}$; 0,298 partes de anhidrita en 100 partes de agua, a $0^{\circ} \mathrm{C}$, y 0,1619 , a $100^{\circ} \mathrm{C}$ ) y solubles en ácidos minerales diluidos, como es el $\mathrm{HCl}$ diluido.

La solubilidad en agua, de estos compuestos, se incrementa en presencia de sales disueltas que aporten iones no comunes, como son $\mathrm{NaCl}, \mathrm{MgCl}_{2}, \mathrm{KNO}_{3}, \mathrm{NaNO}_{3}, \mathrm{Mg}\left(\mathrm{NO}_{3}\right)_{2}$, $\mathrm{NH}_{4} \mathrm{NO}_{3}$, etc. (efecto salino) y disminuye cuando los iones son comunes, como sucede con las sales $\mathrm{CaCl}_{2}, \mathrm{Ca}\left(\mathrm{NO}_{3}\right)_{2}, \mathrm{Na}_{2} \mathrm{SO}_{4}, \mathrm{~K}_{2} \mathrm{SO}_{4}, \mathrm{CaO}$, etc. (efecto del ion común).

Cuando el yeso se somete a la acción del calor, a temperaturas comprendidas entre $128^{\circ} \mathrm{C}$ y $163^{\circ} \mathrm{C}$, pierde aproximadamente $3 / 4$ partes del agua de cristalización, dando el producto conocido con el nombre de hemihidrato $\left(\mathrm{CaSO}_{4} \cdot 1 / 2 . \mathrm{H}_{2} \mathrm{O}<>2 \mathrm{CaSO}_{4} \cdot \mathrm{H}_{2} \mathrm{O}\right)$, el cual se encuentra mezclado con una determinada cantidad de yeso y de anhidrita soluble. A temperaturas superiores se transforma en an'idrita $\left(\mathrm{CaSO}_{4}\right)$ y a $1.200^{\circ} \mathrm{C}$ experimenta la disociación térmica, según:

$$
\mathrm{CaSO}_{4} \text { (sólido) }+ \text { calor }\left(\sim 1.200^{\circ} \mathrm{C}\right) \rightarrow \mathrm{CaO} \text { (sólido) }+\mathrm{SO}_{3} \text { (gas) }
$$

Esta temperatura puede disminuir si hay ciertas sustancias extrañas.

Las impurezas, que normalmente tiene el yeso, afectan a los valores correspondientes al residuo insoluble en una disolución acuosa da $\mathrm{HCl}$ y a la pérdida por calcinación. En efecto, los materiales de naturaleza arcillosa no son solubles en la disolución de dicho ácido y parcialmente solubles los de naturaleza margosa. Los productos a base de calizas, dolomías o margas experimentan por la acción del calor (a temperaturas de 600 a $900^{\circ} \mathrm{C}$ ) la disociación térmica, afectando a los valores de la pérdida de masa por calcinación.

\section{Escoria siderúrgica}

Por escoria siderúrgica se entiende, según el Pliego RC-75, el producto granulado obtenido por enfriamiento brusco de la ganga, modificada en el proceso resultante del tratamiento de los minerales de hierro en los hornos altos.

Las partículas de escoria granulada, de estructura vítrea en gran proporción, están formadas por compuestos cálcicos, normalmente silicatos y aluminosilicatos, solubles en disoluciones acuosas de ácido clorhídrico; prácticamente, las escorias granuladas no dejan residuo insoluble en ácidos.

Las escorias siderúrgicas tienen, además, otros compuestos que presentan propiedades reductoras. Estos compuestos por regla general no existen en los clínkeres normalmente fabricados, aun cuando a veces aparecen en pequeña cantidad, sobre todo en los clínkeres obtenidos en hornos verticales. Dentro de estos compuestos, también solubles en disoluciones acuosas de $\mathrm{HCl}$, cabe resaltar a los sulfuros que, por regla general, no se encuentran en el clínker, ni en el yeso, ni en las puzolanas, y a los compuestos de manganeso (II), hierro (II), etc.

Las escorias siderúrgicas presentan contenidos elevados de compuestos de magnesio (II), en cantidad mayor que en el clínker, formando parte de silicatos y como espinela, a veces se presenta como periclasa; su estado, tanto físico como mineralógico, es distinto al del $\mathrm{MgO}$ activo por lo que, normalmente, no presentan problemas de tipo expansivo. 
Los sulfuros, por la acción de los ácidos minerales, se transformars en $\mathrm{H}_{2} \mathrm{~S}$, fácilmente detectable; basándose en esta propiedad se puede poner de manifiesto la presencia de escoria en el cemento, aun en pequeña cantidad. Cuando las escorias se someten a la acción del calor, en atmósfera oxidante y a $900^{\circ}-1.000^{\circ} \mathrm{C}$, experimentan una variación de masa debida a la transformación (oxidación) de los iones que, con números bajos de valencia, se encuentran formando parte de determinados compuestos S (II), $\mathrm{Fe}$ (II), $\mathrm{Mn}$ (II), etc., los cuales dan lugar a otros compuestos $\mathrm{SO}_{4}$ (II), $\mathrm{Fe}_{2} \mathrm{O}_{3}, \mathrm{MnO}_{2}$, etc. de mayor masa, que permanecen en el producto calcinado.

\section{Puzolanas}

El Pliego RC-75 admite como puzolanas aquellos productos naturales que son capaces de fijar cal a la temperatura ambiente, en presencia de agua, para formar compuestos con propiedades hidráulicas.

Por extensión, el término puzolana se aplica también, en el RC-75, a otros productos naturales o artificiales que tienen propiedades análogas, tales como la tierra de diatomeas, las arcillas activadas y las cenizas volantes.

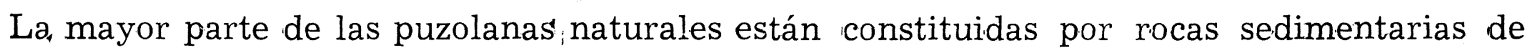
origen volcánico más o menos alteradas; es frecuente que tengan materiales de otro origen en cantidades variables. Dentro de las puzolanas artificiales se incluyen las cenizas volantes y los productos que se obtienen por tratamiento térmico de materias naturales tales como arcillas, esquistos, etc.

Con el nombre de cenizas volantes se conoce a los residuos sólidos, muy finos, procedentes de la combustión de carbón pulverizado que, en las centrales térmicas, son arrastrados fuera del hogar por los gases del proceso. Estos residuos quedan en suspensión en los humos de combustión en forma de partículas fundidas, las cuales solidifican en zonas de temperaturas relativamente bajas.

Los materiales puzolánicos están formados por compuestos silícicos o silicoaluminosos, de composición variable. Del mismo modo, las cenizas volantes, que proceden de las cenizas de los carbones al someterlos en la caldera a una temperatura elevada, están compuestas, en su mayor parte, por productos silícicos o silicoaluminosos, como los de las puzolanas, e inquemados; dicha composición depende del origen y naturaleza de los carbones, así como del tratamiento a que han estado sometidos.

Algunas puzolanas presentan cantidades elevadas de compuestos de magnesio (II); ahora bien, su estado tanto físico como mineralógico es distinto al del óxido de magnesio activo, lo que hace que no presenten problemas de expansión; de aquí que no se limite en el Pliego RC-75 para los cementos siderúrgicos. El contenido de compuestos de magnesio (II) en las cenizas volantes, expresado como $\mathrm{MgO}$, es, por regla general, menor del $5 \%$.

Los compuestos de magnesio que existen en los materiales puzolánicos y en las cenizas volantes son, por regla general, difícilmente solubles en ácidos minerales.

Tanto los productos de los materiales puzolánicos, como los de las cenizas volantes son, en su mayor parte, poco solubles en disoluciones acuosas de $\mathrm{HCl}$. El residuo insoluble en $\mathrm{HCl}$, tratado posteriormente con una disolución de $\mathrm{NaOH}$, de las puzolanas naturales está comprendido en un entorno amplio (15 a $50 \%$ ), así como el de las cenizas volantes (50 a $85 \%)$.

Cuando las puzolanas naturales se someten a la acción del calor experimentan una disminución de masa variable comprendida entre 2 y $15 \%$, debida, fundamentalmente, a la eliminación del agua de constitución de los compuestos que la integran. 
Las cenizas volantes también experimentan una pérdida de masa que oscila del 1 al $15 \%$, producida por la eliminación del agua de humedad residual y por la oxidación de los restos de inquemados que, a la temperatura de 700 a $800^{\circ} \mathrm{C}$ en atmósfera oxidante, se transforman en $\mathrm{CO}_{2}$.

Por otra parte, los compuestos a base de Fe (II), de Mn (II) y de S (II), que se encuentran en las cenizas volantes, dan otros cuerpos, durante la calcinación, de mayor masa que influyen en el resultado final.

\section{ESTUDIO CRITICO DE LOS METODOS DE ENSAYO}

Los métodos de ensayo, descritos en los puntos 8.3, 8.13 y 8.4 del apartado 8 del Pliego RC-75, para determinar el residuo insoluble en ácido clorhídrico, el óxido de magnesio y el trióxido de azufre en los cementos portland, portland con adiciones activas, siderúrgicos (en estos cementos no se especifica el contenido de $\mathrm{MgO}$ ) y puzolánicos (solamente se especifica el contenido de $\mathrm{SO}_{3}$ ), son los mismos. La técnica de trabajo para hallar la pérdida al fuego, o pérdida por calcinación, que se incluye en el punto: 8.2 del apartado 8 de dicho Pliego, se aplica a los cementos portland, portland con adiciones activas y puzolánicos; para los cementos siderúrgicos se utiliza la técnica especificada en el punto 8.18 (determinación del agua y bióxido de carbono) deJ mencionado apartado.

La solubilidad de los silicatos en ácidos es función de la basicidad del catión; así, por ejemplo, los silicatos de sodio son solubles en agua, los de calcio son poco solubles en agua pero fácilmente descompuestos por ácidos minerales diluidos y los de aluminio difícilmente atacados por ácidos. La inmensa mayoría de los silicatos naturales, lo mismo que muchos artificiales, no pueden descomponerse completamente por tratamientos ácidos; sin embargo, cuando se disgregan, se funden o se someten a un calentamiento fuerte con $\mathrm{Na}_{2} \mathrm{CO}_{3}$ ó $\mathrm{NaOH}$, sólidos o en disolución, se transforman en compuestos sódicos atacables por los ácidos.

El residuo insoluble en ácido clorhídrico se puede determinar por dos procedimientos. En el primero se obtiene por ataque de la muestra del cemento, objeto de estudio, con una disolución diluida de ácido clorhídrico para evitar, al máximo, la precipitación del ácido silícico que se encuentra en la disolución; a continuación, el residuo, una vez filtrado, se trata con una disolución de carbonato de sodio, en caliente, con el fin de llevar a disolución las trazas de sílice que hubiesen precipitado y por último, se filtra, se seca, se calcina y se pesa el mencionado residuo.

En el segundo método, el residuo insoluble en ácido clorhídrico, se determina por ataque de la muestra del cemento con una disolución diluida de ácido clorhídrico, filtrado y tratado del residuo con una disolución, a ebullición, de hidróxido de sodio ; a continuación, se filtra, se seca, se calcina y se pesa el residuo.

El residuo insoluble en ácido clorhídrico y carbonato de sodio o hidróxido de sodio está formado por los compuestos no solubles en los reactivos señalados, que no reaccionaron en el proceso de fabricación del clínker o que pueden existir en el yeso como impureza $\mathrm{y}$, principalmente, por los que se encuentran en la adición activa, de un modo especial en las puzolanas, tanto naturales como artificiales, de aquí que, en estos casos, se eleve el contenido especificado en el Pliego RC-75 hasta $8 \%$ para los cementos portland con adiciones activas fabricados a base de puzolana; no se limite si la adición es de ceniza volante y no se fije en los cementos puzolánicos (PUZ-I y PUZ-II).

La determinación del residuo insoluble en ácido clorhídrico y carbonato de sodio o hidróxido de sodio es la menos segura de las utilizadas en el análisis químico de los cementos, 
ya que los silicatos no solubles en ácidos minerales se atacan por las disoluciones concentradas, a ebullición, tanto de carbonato de sodio como de hidróxido de sodio transformándolos en los correspondientes silicatos de sodio solubles en agua. La cantidad obtenida depende del grado de ataque, que es función, para una misma muestra, de la clase y cantidad de reactivos utilizados, tiempo y temperatura de digestión.

El contenido de magnesio (II) se determina gravimétricamente por pesada del pirofosfato de magnesio (punto 8.13, apartado 8 del Pliego RC-75); para ello se precipita el magnesio (II) al estado de fosfato de amonio y magnesio $\left(\mathrm{MgNH}_{4} \mathrm{PO}_{4}\right)\left(^{*}\right)$, una vez llevado a disolución por ataque de la muestra con una disolución acuosa de ácido clorhídrico y eliminados los iones Al (III), Fe (III), Ti (IV) y Ca (II). A continuación, se filtra, se lava, se seca, se calcina el precipitado, obteniéndose el mencionado pirofosfato $\left(\mathrm{Mg}_{2} \mathrm{P}_{2} \mathrm{O}_{7}\right)$, y se pesa.

La precipitación del fosfato de amonio y magnesio, con una $\mathrm{pK}=12,6$, implica varios equilibrios químicos dadas las características de los distintos iones; por una parte, el correspondiente a la reacción de precipitación y, por otra, los de las reacciones de hidrólisis de los iones $\mathrm{NH}_{4}$ (I), ácido conjugado fuerte, y $\mathrm{PO}_{4}$ (III), base conjugada fuerte, por lo que hay que fijar el $\mathrm{pH}$ del medio para eliminar, en lo posible, dichas reacciones, evitar que precipite el $\mathrm{Mg}(\mathrm{OH})_{2}$ y conseguir una fase sólida que responda a la composición teórica de dicho fosfato. La solubilidad del $\mathrm{MgNH}_{4} \mathrm{PO}_{4}\left(8,6 \times 10^{-3} \mathrm{~g} / l\right.$, a $\left.20^{\circ} \mathrm{C}\right)$ aumenta porque se hidroliza fácilmente; la adición de $\mathrm{NH}_{4} \mathrm{OH}\left(\mathrm{NH}_{3}+\mathrm{H}_{2} \mathrm{O}\right)$ o de sales amónicas hace menor esta tendencia.

El fosfato de amonio y magnesio forma con facilidad disoluciones sobresaturadas y, aun cuando exista una pequeña cantidad de fase sólida, el equilibrio de solubilidad se alcanza lentamente; por ello conviene dejar reposar la disolución, al menos, cuatro horas (mejor toda la noche), en frío, antes de separar dicha fase por filtración.

$\mathrm{El} \mathrm{Mg}$ (II) puede formar otros fosfatos poco solubles, como es el $\mathrm{Mg}_{3}\left(\mathrm{PO}_{4}\right)_{2}$ con una $\mathrm{pK}=27,2$, que al calcinarlos darían compuestos distintos al $\mathrm{Mg}_{2} \mathrm{P}_{2} \mathrm{O}_{7}$, obteniéndose resultados erróneos. Estos fenómenos, siguiendo las condiciones de precipitación, llegan a anularse al conseguir que precipite el fosfato de amonio y magnesio; sin embargo, es difícil evitar la coprecipitación, debido a las numerosas sustancias que puede arrastrar.

La determinación exacta del contenido de Mg (II) haciendo una sola precipitación de fosfato de amonio y magnesio lleva consigo, en gran parte, los errores señalados, a menos que se conozca previamente, de un modo aproximado, la cantidad de Mg (II) y las cantidades de reactivos utilizadas sean las apropiadas, de modo que pueda evitarse la coprecipitación de otros fosfatos. En los análisis sistemáticos, donde es necesario realizar diversas operaciones para separar otros cationes antes de precipitar el $\mathrm{Mg}$ (II), se produce la acumulación de iones en la disolución, p.e. oxalato y de un modo especial amonio, procedentes de los reactivos utilizados. El magnesio, en estas condiciones, puede precipitar cuantitativamente la primera vez, aun con otros fosfatos; a pesar de ello, la cantidad de $\mathrm{Mg}$ (II) se puede determinar con precisión, basta con disolver el precipitado con $\mathrm{HCl}$ diluido y volver a precipitar con $\mathrm{NH}_{4} \mathrm{OH}\left(\mathrm{NH}_{3}+\mathrm{H}_{2} \mathrm{O}\right)$ y con una pequeña cantidad, la necesaria, de $\left(\mathrm{NH}_{4}\right)_{2} \mathrm{HPO}_{4}$ ó $\left(\mathrm{NH}_{4}\right)_{3} \mathrm{PO}_{4}$ para disminuir la solubilidad del $\mathrm{MgNH}_{4} \mathrm{PO}_{4}$. De este modo precipita dicho fosfato $\mathrm{y}$ se evita la coprecipitación mencionada anteriormente.

Por otra parte, se ha de señalar que la calcinación del $\mathrm{MgNH}_{4} \mathrm{PO}_{4}$ para obtener $\mathrm{Mg}_{2} \mathrm{P}_{2} \mathrm{O}_{7}$ se efectúa mejor a la temperatura de $1.100^{\circ} \mathrm{C}$, temperatura fijada en determinadas normas, en un horno eléctrico; el pirofosfato de magnesio pierde peso, de un modo suave, a $1.200^{\circ} \mathrm{C}$ y llega, lentamente, a peso constante a $1.000^{\circ} \mathrm{C}$.

(*) Según las condiciones de precipitación puede hacerlo como $\mathrm{MgNH}_{4} \mathrm{PO}_{4} \cdot 6 \mathrm{H}_{2} \mathrm{O}$ ó como $\mathrm{MgNH}_{4} \mathrm{PO}_{4} \cdot \mathrm{H}_{2} \mathrm{O}$. 
La calcinación del $\mathrm{MgNH}_{4} \mathrm{PO}_{4}$ se debe hacer en atmósfera oxidante para evitar la reducción del $\mathrm{Mg}_{2} \mathrm{P}_{2} \mathrm{O}_{7}$, sobre todo si el carbón del papel de filtro está en contacto con el. precipitado a alta temperatura; por ello, éste debe incinerarse a la temperatura más baja posible o por separado con adición de una o dos gotas de $\mathrm{HNO}_{3}$ concentrado, como hace la Recomendación ISO/R 680-1968 cuando la cantidad de precipitado es apreciable, en cuyo caso se calcina a $1.000^{\circ} \mathrm{C}$ durante 20 minutos.

La norma ASTM Designation: C 114-69 (Reapproved 1976) utiliza la doble precipitación del $\mathrm{Mg}$ (II), como $\mathrm{MgNH}_{4} \mathrm{PO}_{4}$, para determinar su contenido en el cemento portland; el precipitado se calcina a $1.100^{\circ} \mathrm{C}$ hasta constancia de peso, tomando las precauciones oportunas para eliminar el papel de filtro y evitar la reducción del precipitado de fosfato.

Como se ha señalado en el apartado anterior, determinados compuestos de magnesio (II) de los distintos componentes de la mezcla son solubles en ácido clorhídrico diluido; de aquí que por medio de esta técnica de trabajo se determine el contenido de $\mathrm{Mg}$ (II) en el cemento, procedente de los compuestos solubles en $\mathrm{HCl}$. Dado que un contenido de $\mathrm{MgO}$ libre (mayor del $5 \%$ ) en el clínker de cemento portland da lugar a fenómenos expansivos, éste únicamente se limita, en el Pliego RC-75, para los cementos portland (P) y para los cementos portland con adiciones activas (PA); sin embargo, las normas de determinados países fijan dichoi contenido de $\mathrm{MgO}$ sólo en el clínker de cemento portland.

El 1. ${ }^{\text {er }}$ anteproyecto de norma CEN/TC 51 N 121 F (1977-12-23) relativo al "Análisis químico de los cementos" describe un método de referencia y otro optativo, ambos complexométricos, para determinar el contenido de Mg (II) en la disolución de la muestra de cemento, obtenida por disgregación con peróxido de sodio o por ataque con ácido clorhídrico y cloruro de amonio, una vez separado el dióxido de silicio. El primero utiliza como reactivo ácido trans-diamino 1,2 ciclohexano-N,N,N',N'-tetraacético (DCTA) y el segundo sal disódica dihidratada del ácido etilen-diamino-tetraacético (EDTA); ambos métodos emplean el mismo indicador, azul de metiltimol con nitrato de potasio. Los puntos de equivalencia se determinan espectrofotométricamente.

Tanto en el método de referencia como en el optativo se determina, previamente, el contenido de $\mathrm{Ca}$ (II) por complexometría con EGTA (ácido etilenglicol-bis-amino-2-etil-N,N, N', N'-tetraacético) y murexido con cloruro de sodio como indicador en el primer método (de referencia) y con EDTA y el mismo indicador en el segundo método (optativo); los puntos de equivalencia se hallan espectrofotométricamente. El magnesio se determina con DCTA para lo cual se enmascara el calcio con EGTA en el método de referencia; eil calcio con EDTA, por una parte, y la suma calcio y magnesio, por otra, en el método optativo, con los indicadores reseñados.

El mencionado anteproyecto admite, cuando no se dispone del equipo espectrofotométrico, la determinación visual del punto de equivalencia; en este caso se emplean como indicadores para el $\mathrm{Mg}$ (II) el azul de metiltimol con nitrato de potasio, en el método de referencia, y púrpura de ftaleína con cloruro de sodio, en el-método optativo. El Ca (II) se determina también previamente con EGTA y el indicador mixto calceína + azul de metiltimol con nitrato de potasio en el método de referencia y con EDTA y calcon con sulfato de sodio en el método optativo.

Cuando en la valoración se utiliza como reactivo la complexona EDTA, se enmascaran los cationes $\mathrm{Al}$ (III), Fe (III) y Ti (IV) con trietanolamina para evitar su interferencia; de esta forma sólo el Ca (II) y el $\mathrm{Mg}$ (II) forman los complejos correspondientes con EDT'A, por lo que se pueden determinar sin separar los cationes señalados en primer lugar.

El contenido de sulfatos en los cementos, según el Pliego RC-75, se determina gravimétricamente; para ello los iones sulfato, que se encuentran en los filtrados ácidos procedentes de la determinación del residuo insoluble, se precipitan, a ebullición, con una disolución de cloruro de bario. El precipitado obtenido de sulfato de bario se filtra, se lava con agua, se seca, se calcina al rojo y, por último, se pesa como $\mathrm{BaSO}_{4}$. 
Otras normas, entre las que se pueden citar las siguientes: ASTM Designation: C 114-69 (Reapproved 1976), ISO/R 680-1968 y 1. ${ }^{\mathrm{er}}$ anteproyecto CEN/TC $51 \mathrm{~N} 121 \mathrm{~F}$ (1977-12-23), utilizan para realizar la determinación del contenido de sulfatos la disolución procedente del ataque de una muestra de cemento con una disolución de ácido clorhídrico, una vez que se ha separado el residuo insoluble. La mencionada norma ASTM admite, además, la disolución obtenida en la determinación del residuo insoluble, técnica que también emplea la norma DIN 1 164, Blatt 3 (April 1967).

La solubilidad del $\mathrm{BaSO}_{4}$ en agua es muy pequeña, así como su variación con la temperatura; en efecto, a $25^{\circ} \mathrm{C}$ es $0,25 \mathrm{mg} / 100 \mathrm{ml}$ de disolución y $0,39 \mathrm{mg}$, a $100^{\circ} \mathrm{C}$. Los ácidos minerales aumentan la solubilidad.

La presencia de una pequeña cantidad de ácido clorhídrico, en el medio en donde tiene lugar la precipitación del $\mathrm{BaSO}_{4}$, evita que precipiten otros iones [bien como sales de bario (II), $\mathrm{Ba}(\mathrm{OH})_{2}$, o como sulfatos (II)] los cuales forman compuestos poco solubles en medio neutro o básico; por otra parte, el medio clorhídrico promueve la formación de un precipitado denso de $\mathrm{BaSO}_{4}$ que sedimenta más rápido. La concentración de ácido clorhídrico que puede usarse en el medio en donde va a tener lugar la precipitación está limitada por la solubilidad del $\mathrm{BaSO}_{4}$; sin embargo, la solubilidad del precipitado en un medio con una acidez clorhídrica $\sim 0,05 \mathrm{~N}(\mathrm{pH} \sim 1,3)$ disminuye en presencia de un exceso de cloruro de bario disuelto. Así, el 1 er $^{\mathrm{er}}$ anteproyecto CEN/TC 51N 121F (1977-12-23) fija el pH de la disolución clorhídrica para la precipitación entre 1 y 1,5.

$\mathrm{El} \mathrm{BaSO}_{4}$ es un precipitado blanco, pulverulento, insoluble en los ácidos minerales, incluso a ebullición; es algo soluble en los ácidos concentrados $\mathrm{HCl}$ o $\mathrm{HNO}_{3}$ y soluble en $\mathrm{H}_{2} \mathrm{SO}_{4}$ concentrado caliente, por lo que no es factible purificarlo por reprecipitación.

$\mathrm{La}$ precipitación del $\mathrm{BaSO}_{4}$ se debe hacer en caliente, para obtener un precipitado que filtre fácilmente, con una disolución diluida de cloruro de bario añadida poco a poco con el fin de evitar la posible coprecipitación; cuando se ha completado la precipitación es necesario añadir un exceso de la disolución de cloruro de bario para disminuir la solubilidad del $\mathrm{BaSO}_{4}$. El tamaño de las partículas de $\mathrm{BaSO}_{4}$ se incrementa cuando se deja en digestión durante un período de tiempo de 12 a 24 horas, a una temperatura inferior, justamente, a la de ebullición, teniendo cuidado de que no se produzca una concentración; si se desea una determinación rápida se puede reducir el tiempo de digestión a 3-4 horas, aun cuando el resultado obtenido puede ser ligeramente inferior. Estos períodos de tiempo son los especificados en las normas ASTM Designation: C 114-69 (Reapproved 1976), ISO/R 680-1968 y en el 1 er $^{\text {er }}$ anteproyecto CEN/TC 51N 121F (1977-12-23); el Pliego RC-75 señala como tiempo de digestión 12-24 horas y la norma DIN 1164, Blatt 3, (April 1967) 2 a 3 horas.

El sulfato de bario puro experimenta la discciación térmica cuando se calienta a $1.400^{\circ} \mathrm{C}$ al aire; sin embargo, a temperaturas superiores a $900^{\circ} \mathrm{C}$ pueda sufrir una descomposición parcial en el caso de que existan vestigios de impurezas, tales como hierro o sílice, en cuyo caso se puede producir una pérdida de $\mathrm{SO}_{3}$ a $1.000^{\circ} \mathrm{C}$.

Cuando el precipitado de $\mathrm{BaSO}_{4}$ se calienta fuertemente en presencia del carbón formado al quemar el papel de filtro se reduce con facilidad a BaS; esta posible reducción se puede eliminar incinerando lentamente el papel de filtro, evitando que forme llama, a una temperatura inferior $\left(600^{\circ} \mathrm{C}\right)$ con acceso libre de aire (atmósfera oxidante) y calcinando posteriormente el precipitado. También se pueden adicionar unas gotas de ácido sulfúrico después de quemar el papel de filtro para transformar el sulfuro en sulfato.

La calcinación final del $\mathrm{BaSO}_{4}$ no es necesario hacerla a una temperatura superior a 800 ó $900^{\circ} \mathrm{C}$; así la norma ASTM Designation: „C 114-69 (Reapproved 1976) y la Recomendación ISO/R 680-1968 fijan el entorno de temperaturas $800-900^{\circ} \mathrm{C}$, la norma DIN 1164 , $900 \pm 25^{\circ} \mathrm{C}$ y el $1 .^{\text {er }}$ anteproyecto CEN/TC $51 \mathrm{~N} 121 \mathrm{~F}, 925 \pm 25^{\circ} \mathrm{C}$. El Pliego RC-75 calcina entre 900 y $1.000^{\circ} \mathrm{C}$. 
Dadas las características del precipitado de $\mathrm{BaSO}_{4}$ es posible preparar, directamente o con ayuda de agentes estabilizadores, suspensiones estables que se pueden utilizar para determinar el contenido de sulfatos por turbidimetría o nefelometría. La norma ASTM Designation: C 114-69 (Reapproved 1976) incluye un método turbidimétrico, opcional, que consiste en medir la turbidez de una disolución en donde se ha precipitado el ion $\mathrm{SO}_{4}$ (II) con $\mathrm{BaCl}_{2} \cdot 2 \mathrm{H}_{2} \mathrm{O}$ cristalizado, de un tamaño de grano dado, con un turbidímetro de Wagner o cualquier otro que tenga el mismo principio. El contenido de sulfato se calcula gráficamente, para lo cual es necesario establecer la correspondiente curva de calibrado a partir de un cemento que tenga una cantidad conocida de $\mathrm{SO}_{4}$ (II).

La principal dificultad de los métodos turbidimétricos o nefelométricos surge de la preparación de la suspensión y especialmente del tamaño de las partículas que es función, entre otras variables, de la concentración de los iones, así como de la relación entre ellas, de la velocidad y forma de realizar la mezcla del tiempo transcurrido antes de efectuar la medida (estabilidad de la suspensión), de la temperatura, de la presencia de otras sustancias, etcétera.

La pérdida al fuego o pérdida por calcinación de una sustancia, o de un conjunto de sustancias, tiene por objeto determinar la variación de masa que experimenta/n cuando se somete/n a la acción de calor, fijando la temperatura, el tiempo de calcinación y la atmósfera de trabajo.

La técnica de trabajo descrita en el Pliego RC-75 para conocer la pérdida al fuego (pérdida por calcinación) de los cementos portland $(\mathrm{P})$, cementos portland con adiciones activas (PA) y puzolánicos (PUZ-I y PUZ-II), consiste en determinar la pérdida de masa, en realidad es la variación de masa como se verá más adelante, que experimenta una muestra de cemento cuando se calienta, en un crisol tapado, al rojo vivo entre $900^{\circ} \mathrm{y}$ $1.000^{\circ} \mathrm{C}$ en una mufla eléctrica o, en su defecto, sobre un buen mechero de gas hasta constancia de peso. La diferencia entre la última pesada (crisol con muestra calcinada) y el peso del crisol con el cemento da la pérdida al fuego correspondiente a la muestra empleada.

Como en las condiciones usuales del ensayo, según RC-75, los cementos siderúrgicos pueden experimentar aumentos de peso debidos a la oxidación del azufre, que contenga al estado de sulfuro, o del hierro divalente existente en la muestra, en este caso, se determina el contenido de agua y de dióxido de carbono ya que, de conformidad con la técnicca cementera, la pérdida por calcinación de los distintos tipos de cemento debe corresponder a la masa de dichos compuestos $\left(\mathrm{CO}_{2}+\mathrm{H}_{2} \mathrm{O}\right)$, eliminados durante la calcinación.

La pérdida por calcinación del cemento portland (P), mezcla de dos componentes (su clínker y regulador del fraguado, según el Pliego RC-75), debe corresponder, teóricamente, a la eliminación del agua de constitución del yeso, suponiendo que sea $\mathrm{CaSO}_{4} \cdot 2 \mathrm{H}_{2} \mathrm{O}$ puro ; sin embargo, los valores experimentales que se obtienen son superiores, debido a la disociación térmica que experimentan las sustancias formadas por la acción del $\mathrm{CO}_{2}$ y de la humedad presentes en la atmósfera (meteorización) sobre los compuestos de la fracción clínker del cemento, fina y activa, y sobre el posible yeso parcialmente deshidratado durante la fabricación del cemento.

La pérdida por calcinación de los cementos con adiciones activas (PA), mezcla de tres -a veces cuatro- componentes (clínker de cemento portland, adición activa y regulador del fraguado) es un proceso aditivo, que corresponde a la variación de masa que experimentan dichos componentes cuando se someten a la acción del calor, siguiendo la técnica de trabajo descrita en el Pliego RC-75.

Tanto el clínker como el yeso experimentan una disminución de masa producida por la eliminación del dióxido de carbono y del agua, como se ha señalado para el caso del cemento portland $(\mathrm{P})$; sin embargo, la posible presencia en las adiciones activas, por una parte, 
de carbono y, por otra, de hierro metálico y de compuestos con iones que se pueden oxidar, como son: sulfuros, hierro (II), manganeso (II), etc., dando otras sustancias de mayor masa, es causa de errores en los valores obtenidos. Así, a la disminución de masa del clínker y del yeso se une la del carbono de las cenizas volantes, al eliminarse como $\mathrm{CO}_{2}$, y el incremento de masa debido a la transformación total o parcial de los iones $\mathrm{S}$ (II) en $\mathrm{SO}_{4}$ (II), $\mathrm{Fe}$ (II) en $\mathrm{Fe}$ (III) y $\mathrm{Mn}$ (II) en $\mathrm{Mn}$ (IV), quedando como $\mathrm{Fe}_{2} \mathrm{O}_{3}$ y $\mathrm{MnO}_{2}$, respectivamente.

Los compuestos de hierro (II), que se encuentran normalmente como $\mathrm{FeO}$, se transforman por la acción del oxígeno del aire en $\mathrm{Fe}_{2} \mathrm{O}_{3}$ experimentando un incremento de masa del $11,13 \%$. El contenido de $\mathrm{FeO}$ en las cenizas volantes es menor del $2 \%$, de aquí que una adición en el cemento del $10 \%$ o del $20 \%$ del peso del cemento portland con adiciones activas, suponga en el caso de una transformación total un incremento de masa menor de 0,02 y $0,04 \%$.

Los compuestos de Mn (II) se presentan en las escorias al estado de sulfuros; su contenido en los productos españoles suele ser menor del 1,6 \% como Mn (II) y del $2 \%$ como $\mathrm{MnO}$. El incremento de masa que corresponde a la transformación del ion $\mathrm{Mn}$ (II) en $\mathrm{MnO}_{2}$ es del $58,24 \%$, por lo que para los cementos con adiciones, de dicha escoria, del 10 y $20 \%$ del peso del cemento portland con adiciones activas, el aumento de masa es menor del 0,09 y $0,18 \%$, respectivamente, suponiendo que todo el $\mathrm{Mn}$ (II) se ha convertido en $\mathrm{MnO}_{2}$.

Los sulfuros metálicos, que de un modo especial existen en la escoria, cuando se someten a la acción del calor, en atmósfera oxidante, sufren una reacción en virtud de la cual los iones $\mathrm{S}$ (II) se convierten en iones $\mathrm{SO}_{4}$ (II), los cuales quedan retenidos en el producto calcinado formando los compuestos cálcicos correspondientes. Este proceso va acompañado de un aumento de masa del $200 \%$ (199,6\%); suponiendo que dichos sulfuros, expresados como S (II), se encuentren en la escoria en una cantidad del $2 \%$ incrementarían la masa del producto 0,4 y $0,8 \%$ para adiciones del 10 y del $20 \%$ del peso del cemento portland con adiciones activas, cuando el $2 \%$ de $\mathrm{S}$ (II) se ha transformado en $\mathrm{SO}_{4}$ (II).

Dado el incremento de masa que experimentan los iones mencionados, así como las cantidades normalmente presentes en las escorias, en las puzolanas naturales y en las cenizas volantes, únicamente se toma en consideración el producido por los sulfuros, por lo que es conveniente introducir un factor de corrección en la pérdida por calcinación o pérdida al fuego experimental para compensar el aumento de masa.

El 1. ${ }^{\mathrm{er}}$ anteproyecto de norma relativa al "Análisis químico de los cementos", CEN/TC 51N 121F (1977-12-23), describe un método de trabajo para conocer la pérdida por calcinación que se aplica a todos los cementos; para ello se determina la pérdida de masa que experimenta una muestra de cemento cuando se somete en un horno eléctrico, a $975 \pm 25^{\circ} \mathrm{C}$, hasta constancia de masa. En aquellos casos en los que se presentan dificultades para obtener dicha constancia de masa, se puede obtener una pérdida por calcinación más precisa determinando el contenido de sulfatos en el producto original y en la muestra calcinada.

En el mencionado anteproyecto se tienen en cuenta los errores causados por la presencia de sulfuros, introduciendo un factor de corrección que es función de la cantidad de S (II) transformado en $\mathrm{SO}_{4}$ (II). La pérdida por calcinación corregida se calcula sumando a la pérdida por calcinación experimental 1,996 veces el $\%$ de $\mathrm{S}$ (II) que se ha oxidado (diferencia entre el $\%$ de S (II) en la muestra original y el de la muestra calcinada) ó 0,8 veces el $\%$ de $\mathrm{SO}_{3}$ que corresponde al de sulfatos procedentes de la oxidación de los sulfuros (diferencia entre el $\%$ de $\mathrm{SO}_{3}$ de la muestra calcinada y el de la muestra original).

La norma ASTM Designation: C 114-69 (Reapproved 1976) describe dos procedimientos para determinar la pérdida por calcinación (a $950 \pm 50^{\circ} \mathrm{C}$ ) de los cementos que contengan escoria ( 25 a $65 \%$ del peso del cemento portland-escoria de horno alto, según la norma 
ANSI/ASTM C 595-76); estos métodos incluyen también una corrección que tiene por. objeto eliminar el error correspondiente a la ganancia de masa debida a la oxidación de los sulfuros. En el primero, método de referencia, a partir del incremento de sulfatos (expresados como $\mathrm{SO}_{3}$ ) que ha tenido lugar durante la calcinación, se calcula la pérdida por calcinación sumando a la experimental 0,8 veces la diferenc:a entre los contenidos de $\mathrm{SO}_{3}$ $(\%)$ de la muestra calcinada y de $\mathrm{SO}_{3}(\%)$ cle la muestra original y en el segundo, método opcional, a partir de la disminución de sulfuros durante la calcinación, se añade a la pérdida por calcinación experimental dos veces la diferencia entre el contenido de $\mathbf{S}$ (II), en $\%$, de la muestra original y el de la muestra calcinada.

La norma DIN 1 164, Blatt 3, 2 (April 1967) determina la pérdida por calcinación de los cementos de escorias sometiendo la muestra a la acción del calor, en un horno tubular, a $750 \pm 25^{\circ} \mathrm{C}$, en crisol de platino y en atmósfera de argón.

Cuando el compunente puzolánico de los cementos portland-puzolana es ceniza volante, la pérdida por calcinación, según la norma ANSt/ASTM C 595-76, se debe hacer en crisol de porcelana a una temperatura situada entre 700 y $800^{\circ} \mathrm{C}$. El contenido de puzolana está comprendido entre 15 y $40 \%$ en peso del cemento puzolánico.

En resumen, a la vista de lo expuesto y teniendo en cuenta los tipos de cementos incluidos en el Pliego RC-75, se ha de tomar en consideración la conveniencia de introducir un factor de corrección en la determinación de la pérdida por calcinación de los cementos portland con adiciones activas (PA), que se puede aplicar a los cementos siderúrgicos (S), con el fin de eliminar el efecto producido por los sulfuros y la de fijar el tipo de crisol, porcelana, que se debe utilizar, así como la temperatura, $700-800^{\circ} \mathrm{C}$, en la técnica de trabajo correspondiente para realizar la pérdida por calcinación de los cementos con cenizas volantes (PA y PUZ-II).

El factor de corrección se puede determinar a partir del incremento de la cantidad de sulfatos en la muestra calcinada (caso $a$ ) o de la disminucióri de sulfuros (caso b) durante el proceso de calcinación.

\section{Caso a. Corrección debida al incremento de sulfatos durante la calcinación}

Como se ha señalado anteriormente, los sulfuros presentes en la muestra de cemento con escorias, durante la calcinación a $900-1.000^{\circ} \mathrm{C}$ en atmósfera oxidante, se transforman total o parcialmente en los sulfatos correspondientes, estables en eỉ entorno mencionado de tem. peraturas.

El incremento de sulfatos, expresados en $\%$ en peso, que ha tenido lugar es igual a:

$\% \mathrm{SO}_{4}$ (II) del cemento calcinado - \% $\mathrm{SO}_{4}$ (II) del cemento sin calcinar; o lo que es lo mismo, teniendo en cuenta que normalmente se expresan como trióxido de azufre:

Incremento $\% \mathrm{SO}_{3}$ (que proceden de la oxidación de los sulfuros, $=\Delta, \% \mathrm{SO}_{3}=\% \mathrm{SO}_{3}$ del cemento calcinado - $\% \mathrm{SO}_{3}$ del cemento sin calcinar.

$\mathrm{El}$ incremento de masa de cada ion S (II) $<>\mathrm{SO}_{4}$ (II) $<>\mathrm{SO}_{3}$, corresponde a $4 \times$ peso atómico-gramo del oxígeno $=4 \times 15,9994 \mathrm{~g}=63,9976 \mathrm{~g}$; es decir, es igual a la masa del oxígeno, fijado por los iones sulfuro, luego el incremento de masa, en tanto por ciento en peso, referido al $\Delta, \% \mathrm{SO}_{3}$ con un peso-molecular gramo $=80,062$, será:

$\Delta$ masa, $\%=\Delta, \% \mathrm{SO}_{3} \times \frac{63,998 \mathrm{~g}}{80,062 \mathrm{~g}}=\Delta, \% \mathrm{SO}_{3} \times 0,799 \sim \Delta, \% \mathrm{SO}_{3} \times 0,8$.

y la pérdida por calcinación corregida (P. C. corregida) será igual a: 
pérdida por calcinación experimental (P. C.) + incremento de masa (masa del oxígeno fijardo), o lo que es lo mismo:

P.C. corregida $=$ P.C. $+0,8 \times \Delta, \% \mathrm{SO}_{3}$.

\section{Caso b. Corrección debida a la disminución de sulfuros durante la calcinación}

Como se ha mencionado, parte de los sulfuros que se encuentran en la muestra de cemento con escorias sin calcinar se transforman, durante la calcinación, en los sulfatos correspondientes, experimentando una disminución parcial o total, según el avance de la reacción.

Por cada ion sulfuro, peso atómico-gramo $=32,064 \mathrm{~g}$, que se oxida se produce un incremento de masa que corresponde a la masa de cuatro átomos de oxígeno $=4 \times$ peso atómico-gramo del oxígeno $=63,9976 \mathrm{~g}$, ya que $\mathrm{S}$ (II) $<>\mathrm{SO}_{4}$ (II); luego el incremento de masa, en tanto por ciento en peso, será:

$$
\Delta \text { masa, } \%=\% \text { sulfuros oxidados } \times \frac{63,998 \mathrm{~g}}{32,064 \mathrm{~g}}
$$

$=\%$ sulfuros oxidados $\times 1,996 \sim \% \mathrm{~S}$ (II) oxidados $\times 2$,

en donde:

$\%$ sulfuros oxidados $=\% \mathrm{~S}$ (II) de la muestra sin calcinar - \% S (II) de la muestra calcinada.

Luego, la pérdida por calcinación corregida (P.C. corregida) será igual a:

pérdida por calcinación experimental (P.C.) + incremento de masa (masa del oxígeno fijado),

o lo que es lo mismo:

P.C. corregida $=$ P.C. $+1,996 \times \%$ S (II) oxidados $\sim$ P.C. $+2 \times \%$ S (II) oxidados.

A continuación, se expone un caso teórico en donde se estudia la influencia que ejercen los sulfuros, presentes en una escoria utilizada para fabricar cemento portland con adiciones activas, sobre la pérdida por calcinación.

En la tabla 3 se incluyen las características químicas de los tres componentes (clínker portland, yeso y escoria siderúrgica) a partir de los cuales se preparan cuatro cementos, uno portland $y_{i}$ tres portland con adiciones activas, que responden a la composición química que figura, en la tabla 4 . La adición ha sido 10, 15 y $20 \%$, en peso, de escoria con relación al peso del cemento PA.

Se ha supuesto que la cantidad de sulfuros en la escoria siderúrgica, expresados como $\mathrm{S}$ (II), es $2 \%$ y, por lo tanto, en cada cemento PA existen, teóricamente, 0,2-0,3 y 0,4\%, como se señala en la tabla 5. Los sulfatos correspondientes a la transformación total de dichos sulfuros durante la calcinación son $0,6-0,9$ y $1,2 \%$, la cantidad total en los cementos calcinados, espresados como $\mathrm{SO}_{3}$, sería 4,4-4,7 y 4,8 \%, respectivamente, en vez de 3,9-3,9 y $3,8 \%$ que hubiesen sido en el caso de no existir sulfuros.

En la tabla 5 se puede observar el incremento de masa que experimentarían los cementos mencionados, debido a la masa del oxígeno fijado por losi iones sulfuro; las correcciones que se deben introducir en la pérdida por calcinación experimental, expresadas con una cifra decimal, obtenidas a partir del aumento de la cantidad de $\mathrm{SO}_{4}<>\mathrm{SO}_{3}$, proceden. tes de la oxidación de S (II), o de la cantidad de sulfuros transformados, así como la pérdida por calcinación experimental y la corregida. 
TABLA 3

Composición química del clínker, del yeso y de la escoria.

Valores expresados en \%, en peso, y referidos a la muestra seca.

\begin{tabular}{|c|c|c|c|}
\hline Composición química & Clínker & Yeso & Escoria \\
\hline $\mathrm{SiO}_{2}$ & 20,7 & - & 35,2 \\
$\mathrm{Al}_{2} \mathrm{O}_{3}$ & 5,7 & - & 17,0 \\
$\mathrm{Fe}_{2} \mathrm{O}_{3}$ & 4,3 & - & 0,7 \\
$\mathrm{CaO}$ & 66,1 & 32,5 & 37,3 \\
$\mathrm{MgO}$ & 1,2 & - & 6,3 \\
$\mathrm{SO}_{3}$ & 0,6 & 46,5 & 0,0 \\
P.C. (P.F.) & 0,3 & 21,0 & - \\
S (II) & - & - & 2,0 \\
\hline
\end{tabular}

TAB L A 4

Cementos portland con adiciones activas. Composición química.

\begin{tabular}{|l|c|c|c|c|c|c|c|}
\hline $\begin{array}{c}\text { Caracte- } \\
\text { rísticas } \\
\text { químicas }\end{array}$ & $\begin{array}{c}\text { Clínker } \\
93 \%\end{array}$ & $\begin{array}{c}\text { Yeso } \\
\mathbf{7} \%\end{array}$ & Cemento & $\begin{array}{c}\text { Clínker } \\
\mathbf{8 3} \%\end{array}$ & $\begin{array}{c}\text { Yeso } \\
\boldsymbol{\%}\end{array}$ & $\begin{array}{c}\text { Escoria } \\
\mathbf{1 0} \%\end{array}$ & Cemento \\
\hline $\mathrm{SiO}_{2}$ & 19,3 & - & 19,3 & 17,2 & - & 3,5 & 20,7 \\
$\mathrm{Al}_{2} \mathrm{O}_{3}$ & 5,3 & - & 5,3 & 4,7 & - & 1,7 & 6,4 \\
$\mathrm{Fe}_{2} \mathrm{O}_{3}$ & 4,0 & - & 4,0 & 3,6 & - & 0,1 & 3,7 \\
$\mathrm{CaO}$ & 61,5 & 2,3 & 63,8 & 54,9 & 2,3 & 3,7 & 60,9 \\
$\mathrm{MgO}$ & 1,1 & - & 1,1 & 1,0 & - & 0,6 & 1,6 \\
$\mathrm{SO}_{3}$ & 0,5 & 3,3 & 3,8 & 0,5 & 3,3 & 0,0 & 3,8 \\
P.C. (P.F.) & 0,3 & 1,5 & 1,8 & 0,3 & 1,5 & - & 1,8 \\
\hline
\end{tabular}

\begin{tabular}{|c|c|c|c|c|c|c|c|c|}
\hline $\begin{array}{l}\text { Caracte- } \\
\text { rísticas } \\
\text { químicas }\end{array}$ & $\begin{array}{c}\text { Clínker } \\
78 \%\end{array}$ & $\begin{array}{l}\text { Yeso } \\
\text { y } \%\end{array}$ & $\begin{array}{c}\text { Escoria } \\
15 \%\end{array}$ & Cemento & $\begin{array}{c}\text { Clínker } \\
73 \%\end{array}$ & $\begin{array}{l}\text { Yeso } \\
y \%\end{array}$ & $\begin{array}{c}\text { Escoria } \\
20 \%\end{array}$ & Cemento \\
\hline $\begin{array}{l}\mathrm{SiO}_{2} \\
\mathrm{Al}_{2} \mathrm{O}_{3} \\
\mathrm{Fe}_{2} \mathrm{O}_{3} \\
\mathrm{CaO} \\
\mathrm{MgO} \\
\mathrm{SO}_{3} \\
\text { P.C. (P.F.) }\end{array}$ & $\begin{array}{r}16,1 \\
4,5 \\
3,4 \\
51,6 \\
0,9 \\
0,5 \\
0,2\end{array}$ & $\begin{array}{l}\bar{Z} \\
\overline{2,3} \\
\overline{3,3} \\
1,5\end{array}$ & $\begin{array}{l}5,3 \\
2,6 \\
0,1 \\
5,6 \\
0,9 \\
0,0 \\
-\end{array}$ & $\begin{array}{r}21,4 \\
7,1 \\
3,5 \\
59,5 \\
1,8 \\
3,8 \\
1,7\end{array}$ & $\begin{array}{r}15,1 \\
4,2 \\
3,1 \\
48,3 \\
0,9 \\
0,4 \\
0,2\end{array}$ & $\begin{array}{l}\overline{-} \\
\overline{2,3} \\
\overline{3,3} \\
1,5\end{array}$ & $\begin{array}{l}7,0 \\
3,4 \\
0,1 \\
7,5 \\
1,3 \\
0,0 \\
\end{array}$ & $\begin{array}{r}22,1 \\
7,6 \\
3,2 \\
58,1 \\
2,2 \\
3,7 \\
1,7\end{array}$ \\
\hline
\end{tabular}

T A B L A 5

\begin{tabular}{|l|c|c|c|}
\hline Características químicas & $\mathbf{1 0} \%$ & $\mathbf{1 5} \%$ & $\mathbf{2 0} \%$ \\
\hline $\begin{array}{l}\%, \mathrm{~S} \text { (II) } \\
\%, \mathrm{SO}_{4} \text { (II) }\end{array}$ & $\begin{array}{l}0,2 \\
0,6\end{array}$ & $\begin{array}{l}0,3 \\
0,9 \\
0,4\end{array}$ & $\begin{array}{l}0,4 \\
1,2 \\
0,8\end{array}$ \\
\hline$\%, \mathrm{SO}_{3}$ & 0,5 & 0,75 & 1,0 \\
\hline$\%, \mathrm{SO}_{3} \times 0,8$ & 0,4 & 0,6 & 0,8 \\
$\%, \mathrm{~S}(\mathrm{II}) \times 1,996$ & 0,4 & 0,6 & 0,8 \\
\hline P.C. experimental & 1,4 & 1,1 & 0,9 \\
P.C. corregida & 1,8 & 1,7 & 1,7 \\
\hline
\end{tabular}




\section{RESUMEN}

En el artículo presente se hace un estudio crítico de las técnicas de trabajo que para determinar las características químicas de los cementos portland (P), de los cementos portland con adiciones activas (PA), de los cementos siderúrgicos (S) y de los cementos puzolánicos (PUZ I y PUZ II), se describen en el Pliego da Prescripciones Técnicas Generales para la Recepción de Cementos (RC-75), teniendo en cuenta:

a. La composición de dichos cementos.

b. Las propiedades más importantes de los componentes de los cementos (clínker de cemento portland, regulador del fraguado, escoria siderúrgica, puzolanas naturales y artificiales, incluidas las cenizas volantes), así como su comportamiento frente a disoluciones acuosas de ácido clorhídrico y cuando se someten a la acción del calor.

c. Los fundamentos fisicoquímicos, desde un punto de vista analítico, de los métodos de trabajo que se aplican en cada caso particular.

d. Las precauciones que se deben tomar en las diversas operaciones que normalmente se efectúan (ataque-disolución de la muestra, eliminación de las interferencias producidas por la presencia de determinados iones o compuestos, separación-precipitacióncristalización, filtrado-lavado y purificado de precipitados, secado y calcinación de los mismos) para eliminar las posibles causas de error y obtener resultados fiables.

e. Los procedimientos que se utilizan en otras normas.

Por último y como consecuencia del estudio efectuado, se considera la conveniencia de introducir determinadas modificaciones en los métodos de ensayo descritos, así como ciertos factores de corrección en la determinación de la pérdida por calcinación o pérdida al fuego.

\section{BIBLIOGRAFIA CONSULTADA}

- Alonso, J. L.: Estudio Físico-Químico y Técnico de diversos tipos de cenizas y su empleo como material de construcción. Laboratorio Central de Ensayo de Materiales. Publicación n. ${ }^{\circ}$ 199. Madrid, 1960.

- Ayres, G. H.: Análisis Químico Cuantitativo. Ediciones del Castillo, S. A. Madrid, 1970.

- BenNett, H. and Hawley, W. G.: Methodes of Silicate Analysis. Academic Press. London and New York, 1965.

- Berl-Lunge-D'Ans: Métodos de Análisis Quimico Industrial. Tomos II (primera parte) y III. Editorial Labor, S. A. Barcelona, Madrid, Buenos Aires, Río de Janeiro, 1945-1946.

- Bermejo, F.: Quimica Analítica General Cuantitativa e Instrumental. Facultad de Ciencias. Santiago do Compostela, 1974.

- Blondiau, L.: Dosage néphélometrique de l'anhydride sulfurique dans les ciments. Rev. des Mat. de Const. 1962-1965, 378, (1947); 195-198, 379, (1947); 227-230, 380, (1947); 259-261, 381, (1947) у 284-286, 382, (1947).

- Butterworth, B.: La Brique. Dunod. París.

- Bombled, J. P.: Les essais interlaboratoires conduits par le CERILH. Rev. des Mat.; 103-112, 687, (1947).

- Calleja, J.: Las puzolanas. Ion; 623-638, 340, (1958); 700-713, 341, (1958), 81-90, 343, (1958) y 154-160, 344, (1958).

- Calleja, J.: Cemento Portland: Cálculo e interpretación de datos químicos. Manuales y Normas del Instituto Técnico de la Construcción y del Cemento. Madrid, 1960.

- Calleja, J.; Fernandez Paris, J. M. y Triviño, F.: Métodos complexométricos para el análisis rápido del cemento portland. Manuales y Normas del Instituto Eduardo Torroja de la Construcción y del Cemento. Madrid, 1964.

- Calleja, J.: Criterios sobre normas para cemento Ion; 253-266, 310, (1967).

20 
- Calleja, J.: Consideraciones sobre el cálculo de la composición potencial de los conglomerantes hidráulicos. Materiales de Construcción, Ultimos Avances. 5-26, 125, (1967) y 9-20, 126, (1967).

- Calleja, J.: Las nuevas normas españolas para cemento: Materiales de Construcción, 5-24, 164, (1976).

- Calleja, J.: Código de buena práctica para la utilización de los cementos del Pliego RC-75. Manuales y Normas del Instituto Eduardo Torroja de la Construcción y del Cemento. Madrid, 1977.

- Coronas, J. M.: Estudio crítico de los métodos para el análisis del cementc portland y elección del inétodo apropiado. Laboratorio Central de Ensayo de Materiales de Construcción. Publicación n. ${ }^{\circ} 26$. Ma- $^{2}$ drid, 1945.

- ChaIken, B.: Determination of Ignition Loss in Portland Blast Furnace Slag Cement. ASTM, Bull., 5358, 238, (1959).

- Charlot, G. et Bezier, D.: Méthodes Modernes d'Analyse Quantitative Minerale. Miasson et Cie. París, 1948.

- Degre, M.: Dosage des sulfates dans les ciments avec l'autoanalyseur Technicon. Rev. des Mat. de Const., 36-39, 686, (1974).

- Duva, W. H.: Manual Tecnológico del cemento. Editores Técnicos Asociados, S. A. Barcelona, 1977.

- Duriez, M. et Arrambadi, J.: Nouveau traité de Matériaux de Construction. Dunod. París, 1961-1962.

- Gaspar-Tebar, D.: Arcillas. Instituto Técnico de la Construcción y del Cemento. Madrid, 1951.

- Gaspar-Tebar, D.: Algunas consideraciones sobre la presencia de sulfuros metálicos en los áridos. Materiales de Construcción, 29-42, 159, (1976).

- Gaspar-Tebar, D.: Determinación del contenido de escorias en los cementos siderúrgicos. Materiales de Construcción, 9-29, 162, (1976).

- Gonzalez, F.: Manual de Ensayo de Cementos. Editorial Dossat, S. A. Madrid, 1950.

- Groves, A. W.: Gypsum and Anhydrite. Her Majesty's Stationery Office. London, 1958.

- Groves, A. W.: Silicate Analysis. George Allen and Unwin, Ltd. London, 1951.

- Harmelin, M.: La Thermo-analye (Que sais je?, n. ${ }^{\circ}$ 1.318). Presses Universitaires de France. París, 1968.

- JАков, J.: Guía para el análisis químico de las rocas. Instituto Alonso Barba (CSIC). Madrid, 1944.

- Jeffery, P. G.: Chemical Methods of Rock Analysis. Pergamon Press. Oxford, 1970.

- KEIL, F.: Cemento. Editores Técnicos Asociados, S. A. Barcelona, 1973.

- Kolthoff, I. M. and Elving, Ph. J.: Treatise on Analytical Chemistry; Part. I. Theory and Practice; wolume 11. A. Willey-Interscience Publication, Jonh Willey and Sons. New York, London, Sydney, Toronto, 1975 .

- Kolthoff, I. M.; Sandell, E. B.; Mechan, E. J. y Bruckenstein, S.: Análisis Químico Cuantitativo. Librería y Editorial Nigar, S. R. L. Buenos Aires, 1972.

- LAssieur, A.: Analyse des Silicates. Dunod. París, 1951.

- LEA, F. M.: Investigations on pozzolanas. I. Pozzolanas and Lime-Pozzolana mixex. Building Research. Technical Paper No 27. London, 1940.

- LEA, F. M.: The Chemistry of Cement and Concrete. Edward Arnold. Ltd. Glasgow, 1970.

- Luxan, M. P.: Métodos de valoración cualitativo y cuantitativo de las adiciones de tipo silíceo presentes en el cemento. Cemento-Hormigón, 91-103, 530, 1978.

- Luxan, M. P.: Método de valoración cualitativo y cuantitativo de las adiciones silíceas presentes en el cemento. Tesis Doctoral. Madrid, 1975.

- Massazza, F.: Chimica delle aggiunte pozzolaniche e dei cementi di miscela. Il Cemento, 3-28, 1, (1976).

- Meric, J. P.; LE JEAN, Y. et Caron, J. F.: Analyse elementaire des crus de cimenterie par excitation Laser. Rev. des Mat. 36-42, 684, (1973).

- Miusikas, N.: Détermination des teneurs en constituants secondaires dans un ciment Portland composé. Rev. des Mat., 21-35, 652-653, (1970).

- SAssenscheidt, A.: Die Bestimmung von Kohlenstoff in Rohmehlen und Zementen. Zement-Kalk-Gips, 23-26, 1, (1960).

- Sassenscheidr, A.: Beitrag zur Bestimmung des Glühverlustes von Zementen. Zement-Kalk-Gips, 149-156, 4, (1960).

- Schwarzenbach, G. and Flaschka, H.: Complexometric Titrations. Methuen and Co., Ltd. London, 1969.

- Soria, F.: Puzolanas y cementos puzolánicos. Conferencia pronunciada en Madrid, 1962.

- Soria, F.: Tecnología de los cementos. Universidad de Chile; Instituto de Investigaciones y Ensayo de Materiales. Santiago de Chile, 1976.

- TAylor, H. F. W.: La Química de los cementos. Ediciones Urmo. Bilbao, 1967. 
- Treadwell, F. P.: Tratado de Quimica Analítica. Análisis Cuantitativo. Editor Manuel Marín. Barcelona, 1942

- Vogel, A. I.: A. Text-book of Quantitative Inorganic Analysis. Third Edition. London 1972.

- Voinovitch, I. A.; Debras-Guedon, J. et Louvrier, J.: L'analyse des silicates. Hermann. París, 1962.

- Wischers, G.: Normalización del cemento. Notas y explicaciones respecto a la reelaboración de la norma alemana DIN 1164; redacción junio 1970. Materiales de Construcción; Ultimos Avances, 43-56, 145, (1972) y 67-83, 146. (1972).

\section{NORMAS CONSULTADAS}

- DIN 1164, Blatt 3: Portland-Eisenportland-Hochofen-Trasszement. "Bestimmung der Zusammensetzung". April, 1967.

- ISO R 680-1968: Analyse chimique des ciments. Eléments principaux du Ciment portland. Mars, 1968.

- Anónimo: Cement Standards of the world. Paris, 1968.

- Pliego de Prescripciones Técnicas Generales para la Recepción de Cementos (RC-75): B.O.E., 18197-18213, n. ${ }^{\circ} 206$, (28 agosto 1975) y 18299-18311, n. ${ }^{\circ} 207$, (29 agosto 1975).

- ASTM Designation: C-114-69 (Reapproved 1976): Chemical Analysis of Hydraulic Cement.

- ANSI/ASTM C 595-76: Standard Specifications for Blended Hydraulic Cements.

- NF P 15-301 (Août 1976): Liants Hydrauliques. Definitions, classification et spécifications des ciments.

- CEN/TC 51N 121F: Analyse Chimique des ciments: (1. ${ }^{\text {cr }}$ avant-project de norme présenté par M. Von EUW, responsable du groupe de travail CEN/TC 51/GT 3). 1977-12-23.

- Anónimo: La nouvelle normalisation française des ciments. Centre d'Information de l'Industrie Cimentière. París, septembre 1978. 